\title{
Distributed Fusion Filter for Networked Multi-sensor Systems with Unknown Measurement Interferences and Packet Dropouts
}

\author{
Bo $\mathrm{Qi}^{1, \mathrm{a}}$, Shuli Sun ${ }^{2, \mathrm{~b}}$ \\ ${ }^{1}$ School of Electronic Engineering, Heilongjiang University, Harbin, 150080, China \\ ${ }^{2}$ School of Electronic Engineering, Heilongjiang University, Harbin, 150080, China \\ aemail: qibo_2010@163.com, bemail: sunsl@hlju.edu.cn
}

\begin{abstract}
Keywords: Filter; Networked System; Unknown Interference; Packet Dropout; Linear Unbiased Minimum Variance
\end{abstract}

\begin{abstract}
This paper is concerned with the design of distributed fusion filter for networked systems with unknown measurement interferences and packet dropouts. A Bernoulli distributed random variable is used to depict the phenomenon of packet dropouts. Without any prior information about the interference, a recursive Kalman-type state filter independent of the unknown interferences is designed for each sensor subsystem by applying the linear unbiased minimum variance estimation criterion. Based on the state filters of individual subsystems, the estimation error cross-covariance matrices between any two subsystems are derived. Then, the distributed fusion filter is designed by using the matrix-weighted fusion estimation algorithm in the linear minimum variance sense. Simulation results show the effectiveness of the proposed algorithms.
\end{abstract}

\section{Introduction}

With the rapid development of electronics, communication and computer technologies, networked control systems have been gradually applied to all aspects of productions and lives due to the convenient connection mode and high-speed transmission. The state estimation problems for networked control systems have attracted the interests of many scholars [1-4]. However, the networks bring convenience meanwhile introduce a lot of uncertainties. Due to the limitation of network bandwidths, there are the transmission delays and losses of data.

There are many reports about the state estimation problems for systems with time delays and packet dropouts [5-8]. A linear minimum variance filter dependent on the probability of missing measurements is presented in [5]. The optimal linear estimation problem about multiple packet dropouts is studied in [6]. The missing measurements and time-varying delays existing in uncertain stochastic networks are considered in [7]. Ref. [8] proposes a state filter for networked systems with multiple random delays and packet losses. In addition, the external interferences and syntheses of device failures have effect on the sensor outputs, which results in the uncertainties of measurement outputs. In recent years, the state estimation for systems with unknown inputs has also become a hot research topic [9-11], especially in the application of fault diagnosis. A fault diagnosis method based on the optimal unknown input observer is presented in [12]. Ref. [13] proposes a fault detection filter for linear discrete time-varying systems with multiple packet dropouts. Ref. [14] gives a fusion predictor for multi-sensor systems with missing measurements and unknown measurement interferences, where the computation of the state second-order moment is required.

Based on the results of references above, for the multi-sensor networked systems with unknown measurement interferences and packet dropouts, we present a recursive Kalman-type local state filter independent of unknown interferences for each sensor subsystem based on the linear unbiased minimum variance estimation criterion [15]. Further, the estimation error cross-covariance matrices between any two subsystems are derived. At last, we give the distributed fusion filter weighted by matrices in the linear minimum variance sense. Differently from [14], the computation of the state second-order moment is avoided. 


\section{Problem formulation}

Consider a multi-sensor linear discrete stochastic system with unknown measurement interferences and packet dropouts:

$$
\begin{aligned}
& x(t+1)=\Phi x(t)+\Gamma w(t) \\
& y_{i}(t)=H_{i} x(t)+v_{i}(t)+D_{i} \theta_{i}(t), i=1,2, \cdots, L \\
& z_{i}(t)=u_{i}(t) y_{i}(t), i=1,2, \cdots, L
\end{aligned}
$$

In the type: $x(t) \in R^{n}$ is the state, $y_{i}(t) \in R^{m_{i}}$ is the measured output of the $i$ th sensor, which will be transmitted to the filter through networks, $Z_{i}(t) \in R^{m_{i}}$ is the measurement received by the filter, and $\theta_{i}(t) \in R^{p_{i}}$ signifies the unknown sensor measurement interference. $L$ is the number of sensors. $w(t) \in R^{r}$ and $v_{i}(t) \in R^{m_{i}}$ are the process and measurement noises. $\left\{u_{i}(t)\right\}$ is a known white Bernoulli distributed stochastic sequence taking values 1 and 0 with the probability $\operatorname{Prob}\left\{u_{i}(t)=1\right\}=\alpha_{i}, \operatorname{Prob}\left\{u_{i}(t)=0\right\}=1-\alpha_{i}, \quad 0 \leq \alpha_{i} \leq 1$, and independent of other stochastic variables. If $u_{i}(t)=1$, the measurement of the $i$ th sensor is received during transmissions, otherwise, the filter receives nothing, which means packet dropout. Moreover, $\Phi, \Gamma, H_{i}$ and $D_{i}$ are constant matrices with suitable dimensions.

We will present our main results based on the following assumptions.

Assumption 1: $w(t)$ and $v_{i}(t)$ are uncorrelated white noises with mean 0 and the variances $Q_{w} \geq 0$ and $Q_{v_{i}}>0$.

Assumption 2: The initial state $x(0)$ is uncorrelated with $w(t), v_{i}(t)$ and $u_{i}(t)$, and satisfies

$$
\mathrm{E}\{x(0)\}=\mu_{0}, \mathrm{E}\left\{\left[x(0)-\mu_{0}\right]\left[x(0)-\mu_{0}\right]^{\mathrm{T}}\right\}=P_{0}
$$

where symbol $\mathrm{E}$ denotes the mathematical expectation, $\mathrm{T}$ is the transpose operator.

Assumption 3: $\operatorname{rank}\left[D_{i}\right]=p_{i}<m_{i}, \operatorname{rank}[*]$ denotes the rank of matrix *.

The objectives of this paper are to design the recursive Kalman-type local state filter $\hat{x}_{i}(t)$ by applying the linear unbiased minimum variance estimation criterion based on the received measurements $\left(z_{i}(t), z_{i}(t-1), \cdots, z_{i}(1)\right)$ of the $i$ th sensor and the distributed fusion filter $\hat{x}_{o}(t)$.

\section{Local state filter design}

In this section, since there is not any prior information about the interferences, a recursive Kalman-type local state filter independent of the unknown interference will be designed.

Theorem 1: For system (1)-(3) under Assumptions 1-3, the recursive local state filter is calculated as follows:

$$
\hat{x}_{i}(t+1)=\Phi \hat{x}_{i}(t)+u_{i}(t+1) K_{i}(t+1)\left[z_{i}(t+1)-H_{i} \Phi \hat{x}_{i}(t)\right]
$$

where

$$
\begin{aligned}
& K_{i}(t+1)=\left[\bar{P}_{i}^{\mathrm{T}}(t) H_{i}^{\mathrm{T}}-\Lambda_{i}(t+1) D_{i}^{\mathrm{T}}\right] C_{i}^{-1}(t+1) \\
& \Lambda_{i}(t+1)=\bar{P}_{i}^{\mathrm{T}}(t) H_{i}^{\mathrm{T}} C_{i}^{-1}(t+1) D_{i}\left[D_{i}^{\mathrm{T}} C_{i}^{-1}(t+1) D_{i}\right]^{-1} \\
& C_{i}(t+1)=H_{i} \bar{P}_{i}(t) H_{i}^{\mathrm{T}}+Q_{v_{i}}
\end{aligned}
$$

The filtering error variance matrix is given as

$$
\begin{aligned}
& P_{i}(t+1)=\bar{P}_{i}(t)+u_{i}(t+1) K_{i}(t+1) C_{i}(t+1) K_{i}^{\mathrm{T}}(t+1)-u_{i}(t+1) K_{i}(t+1) H_{i} \bar{P}_{i}(t) \\
& -u_{i}(t+1) \bar{P}_{i}^{\mathrm{T}}(t) H_{i}^{\mathrm{T}} K_{i}^{\mathrm{T}}(t+1)
\end{aligned}
$$

where

$$
\bar{P}_{i}(t)=\Phi P_{i}(t) \Phi^{\mathrm{T}}+\Gamma Q_{w} \Gamma^{\mathrm{T}}
$$

with the initial value $\hat{x}_{i}(0)=\mu_{0}$ and $P_{i}(0)=P_{0}$. 
Proof: (1) When $u_{i}(t+1)=1$, i.e., there is not packet dropout for the ith sensor subsystem, we will design the following recursive Kalman-type local state filter

$\hat{x}_{i}(t+1)=\Phi \hat{x}_{i}(t)+K_{i}(t+1)\left[z_{i}(t+1)-H_{i} \Phi \hat{x}_{i}(t)\right]$

From (1)-(3) and(11), the filtering error equation of the ith subsystem is derived as follows:

$\tilde{x}_{i}(t+1)=x(t+1)-\hat{x}_{i}(t+1)$

$=\left[I-K_{i}(t+1) H_{i}\right] \Phi \tilde{x}_{i}(t)+\left[I-K_{i}(t+1) H_{i}\right] \Gamma w(t)-K_{i}(t+1) v_{i}(t+1)-K_{i}(t+1) D_{i} \theta_{i}(t+1)$

From the unbiasedness $\mathrm{E}\left[\tilde{x}_{i}(t)\right]=0$, we have

$K_{i}(t+1) D_{i}=0$

Substituting (13) into (12) yields

$$
\tilde{x}_{i}(t+1)=\left[I-K_{i}(t+1) H_{i}\right] \Phi \tilde{x}_{i}(t)+\left[I-K_{i}(t+1) H_{i}\right] \Gamma w(t)-K_{i}(t+1) v_{i}(t+1)
$$

Substituting (14) into $P_{i}(t+1)=\mathrm{E}\left[\tilde{x}_{i}(t+1) \tilde{x}_{i}^{\mathrm{T}}(t+1)\right]$ yields the filtering error variance as follows:

$$
\begin{aligned}
& P_{i}(t+1)=\left[I-K_{i}(t+1) H_{i}\right] \Phi P_{i}(t) \Phi^{\mathrm{T}}\left[I-K_{i}(t+1) H_{i}\right]^{\mathrm{T}}+\left[I-K_{i}(t+1) H_{i}\right] \Gamma Q_{w} \Gamma^{\mathrm{T}}\left[I-K_{i}(t+1) H_{i}\right]^{\mathrm{T}} \\
& +K_{i}(t+1) Q_{v_{i}} K_{i}^{\mathrm{T}}(t+1)
\end{aligned}
$$

Arranging and simplifying (15) gives

$$
P_{i}(t+1)=\bar{P}_{i}(t)+K_{i}(t+1) C_{i}(t+1) K_{i}^{\mathrm{T}}(t+1)-K_{i}(t+1) H_{i} \bar{P}_{i}(t)-\bar{P}_{i}^{\mathrm{T}}(t) H_{i}^{\mathrm{T}} K_{i}^{\mathrm{T}}(t+1)
$$

where $\bar{P}_{i}(t)$ and $C_{i}(t+1)$ are defined by (10) and (8), respectively.

Applying the linear unbiased minimum variance estimation criterion [16], under the constraint condition (13) we introduce the auxiliary equation as follows:

$$
J_{i}(t+1)=\operatorname{tr}\left\{P_{i}(t+1)\right\}+2 \operatorname{tr}\left\{\Lambda_{i}^{\mathrm{T}}(t+1) K_{i}(t+1) D_{i}\right\}
$$

Where $\operatorname{tr}\{*\}$ denotes the trace of the matrix $*$. To minimize the performance index $J_{i}(t+1)$, letting $\partial J_{i}(t+1) / \partial K_{i}(t+1)=0$ and using the derivative formula for trace of matrix [15], we have

$$
K_{i}(t+1) C_{i}(t+1)+\Lambda_{i}(t+1) D_{i}^{\mathrm{T}}=\bar{P}_{i}^{\mathrm{T}}(t) H_{i}^{\mathrm{T}}
$$

Combining (13) with (18) gives the matrix equation

$$
\left[\begin{array}{cc}
C_{i}(t+1) & D_{i} \\
D_{i}^{\mathrm{T}} & 0
\end{array}\right]\left[\begin{array}{c}
K_{i}^{\mathrm{T}}(t+1) \\
\Lambda_{i}^{\mathrm{T}}(t+1)
\end{array}\right]=\left[\begin{array}{c}
H_{i} \bar{P}_{i}(t) \\
0
\end{array}\right]
$$

From Assumption 1 and Assumption 3, we find that the inverse of coefficient matrix in (19) exists. Then, we have the solutions (6) and (7).

(2) When $u_{i}(t+1)=0$, i.e., there is packet dropout, since no measurement is available, we will predict based on the estimate at last time, i.e.,

$$
\hat{x}_{i}(t+1)=\Phi \hat{x}_{i}(t)
$$

Then, the estimation error equation is given as

$$
\tilde{x}_{i}(t+1)=x(t+1)-\hat{x}_{i}(t+1)=\Phi \tilde{x}_{i}(t)+\Gamma w(t)
$$

The estimation error variance can be calculated as

$$
P_{i}(t+1)=\Phi P_{i}(t) \Phi^{\mathrm{T}}+\Gamma Q_{w} \Gamma^{\mathrm{T}}
$$

In summary, (11) and (20) yield (5). (16) and (22) yield (9). This proof is completed.

\section{Distributed fusion filter}

Theorem 2: For system (1)-(3) under Assumptions 1-3, according to the case whether the measurement is received by the filter, we discuss the calculation formula for the estimation error cross-covariance matrix as follows:

$$
P_{i j}(t+1)=\left[I-u_{i}(t+1) K_{i}(t+1) H_{i}\right]\left[\Phi P_{i j}(t) \Phi^{\mathrm{T}}+\Gamma Q_{w} \Gamma^{\mathrm{T}}\right]\left[I-u_{j}(t+1) K_{j}(t+1) H_{j}\right]^{\mathrm{T}}
$$

with the initial value $P_{i j}(0)=P_{0}$.

Proof: If the measurements from the $i$ th and $j$ th sensors are both received by the filter, 
$u_{i}(t+1)=1$ and $u_{j}(t+1)=1$. From (14), the filtering error equations are given as

$$
\tilde{x}_{s}(t+1)=\left[I-K_{s}(t+1) H_{s}\right] \Phi \tilde{x}_{s}(t)+\left[I-K_{s}(t+1) H_{s}\right] \Gamma w(t)-K_{s}(t+1) v_{s}(t+1), s=i, j
$$

Substituting (24) into the estimation error covariance matrix $P_{i j}(t+1)=\mathrm{E}\left[\tilde{x}_{i}(t+1) \tilde{x}_{j}^{\mathrm{T}}(t+1)\right]$ yields

$$
P_{i j}(t+1)=\left[I-K_{i}(t+1) H_{i}\right]\left[\Phi P_{i j}(t) \Phi^{\mathrm{T}}+\Gamma Q_{w} \Gamma^{\mathrm{T}}\right]\left[I-K_{j}(t+1) H_{j}\right]^{\mathrm{T}}
$$

If the measurements from one of the $i$ th and $j$ th sensors are received by the filter, without loss of generality, assuming the measurements of the ith sensor are received, $u_{i}(t+1)=1$ and $u_{j}(t+1)=0$. The filtering error equation for the ith sensor is given by (24). From (21), the prediction error equation for the $j$ th sensor is given as

$$
\tilde{x}_{j}(t+1)=\Phi \tilde{x}_{j}(t)+\Gamma w(t)
$$

Substituting (24) and (26) into $P_{i j}(t+1)=\mathrm{E}\left[\tilde{x}_{i}(t+1) \tilde{x}_{j}^{\mathrm{T}}(t+1)\right]$ yields

$$
P_{i j}(t+1)=\left[I-K_{i}(t+1) H_{i}\right]\left[\Phi P_{i j}(t) \Phi^{\mathrm{T}}+\Gamma Q_{w} \Gamma^{\mathrm{T}}\right]
$$

If the measurements from the $i$ th and $j$ th sensors are both lost, $u_{i}(t+1)=0$ and $u_{j}(t+1)=0$. The prediction error equations of two sensors are given by (26). Substituting (26) into $P_{i j}(t+1)=\mathrm{E}\left[\tilde{x}_{i}(t+1) \tilde{x}_{j}^{\mathrm{T}}(t+1)\right]$ yields

$$
P_{i j}(t+1)=\Phi P_{i j}(t) \Phi^{\mathrm{T}}+\Gamma Q_{w} \Gamma^{\mathrm{T}}
$$

Summarizing (25), (27) and (28), we have (23). This proof is completed.

Based on local filters in Theorem 1 and the cross-covariance matrices in Theorem 2, we have the following distributed fusion filter by using the matrix-weighted fusion estimation algorithm in the linear minimum variance sense [16]:

$$
\hat{x}_{o}(t)=\sum_{i=1}^{L} \bar{A}_{i}(t) \hat{x}_{i}(t)
$$

The matrix $\bar{A}_{i}(t), \quad i=1,2, \cdots, L$, are computed by

$$
\left[\bar{A}_{1}(t), \bar{A}_{2}(t), \cdots, \bar{A}_{L}(t)\right]=\left(e^{\mathrm{T}} \Sigma^{-1}(t) e\right)^{-1} e^{\mathrm{T}} \Sigma^{-1}(t)
$$

where $e=[I, I, \cdots, I]^{\mathrm{T}}$ is a matrix with $n L \times n$ dimension, $\Sigma(t)$ is an $n L \times n L$ matrix whose $(i, j)$ th block element is $P_{i j}(t)$. The variance matrix of the distributed fusion filter is

$$
P_{o}(t)=\left(e^{\mathrm{T}} \Sigma^{-1}(t) e\right)^{-1}
$$

Moreover, we have $P_{o}(t) \leq P_{i}(t), i=1,2, \cdots, L$.

\section{Simulation example}

Consider the following tracking system

$$
\begin{aligned}
& x(t+1)=\left[\begin{array}{ll}
1 & T \\
0 & 1
\end{array}\right] x(t)+\left[\begin{array}{c}
T^{2} / 2 \\
T
\end{array}\right] w(t) \\
& y_{i}(t)=H_{i} x(t)+v_{i}(t)+D_{i} \theta_{i}(t), i=1,2,3 \\
& z_{i}(t)=u_{i}(t) y_{i}(t), i=1,2,3
\end{aligned}
$$

Sampling period is $T=0.5$, the observation matrices are $H_{1}=\left[\begin{array}{ll}1 & 2 \\ 0 & 1\end{array}\right], H_{2}=\left[\begin{array}{ll}1 & 0 \\ 2 & 1\end{array}\right], H_{3}=\left[\begin{array}{ll}2 & 1 \\ 1 & 1\end{array}\right]$. The white noise $w(t)$ with variance $Q_{w}=1$ is uncorrelated with white noises $v_{i}(t)$ with variances $Q_{v_{1}}=0.36 I_{2}, Q_{v_{2}}=0.81 I_{2}$ and $Q_{v_{3}}=0.64 I_{2}$, respectively, and $I_{2}$ is a $2 \times 2$ identity matrix. The unknown interferences in the measurement outputs are set as $\theta_{1}(t)=3, \theta_{2}(t)=0.1 t$ and $\theta_{3}(t)=2 \sin (t / 2) . D_{1}=[1,1]^{\mathrm{T}}, D_{2}=[2,1]^{\mathrm{T}}, D_{3}=[1,3]^{\mathrm{T}}$. The initial values are $x(0)=[0,0]^{\mathrm{T}}$ and $P_{0}=0.01 I_{2}$. We take 100 sampling data. Our aim is to design the distributed fusion filter $\hat{x}_{o}(t)$. 


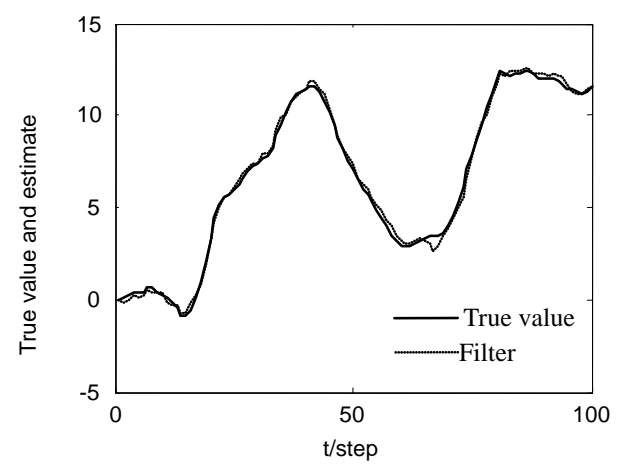

(a) The first-state component

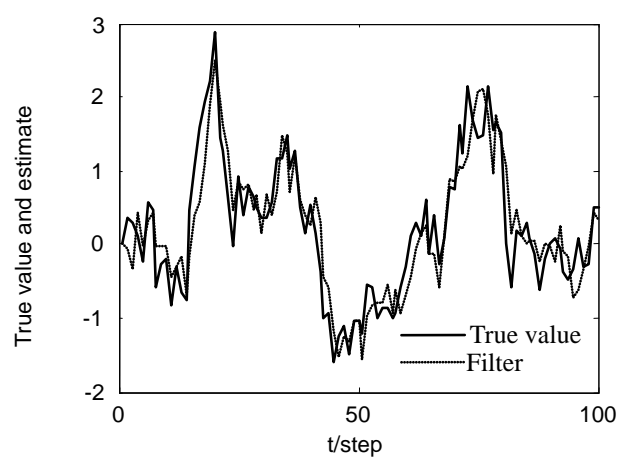

(b) The second-state component

Fig.1. Distributed fusion state filter

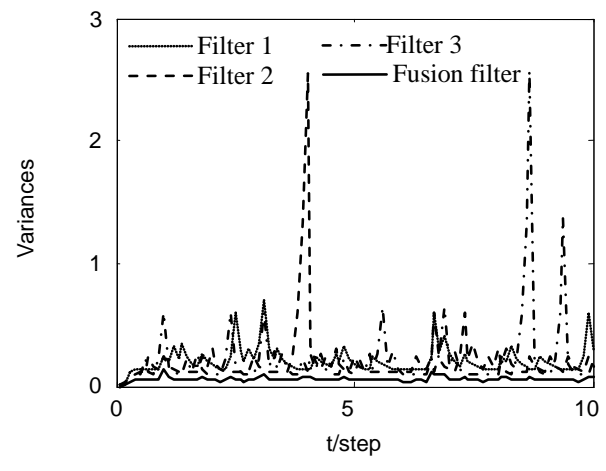

(a) The first-state component

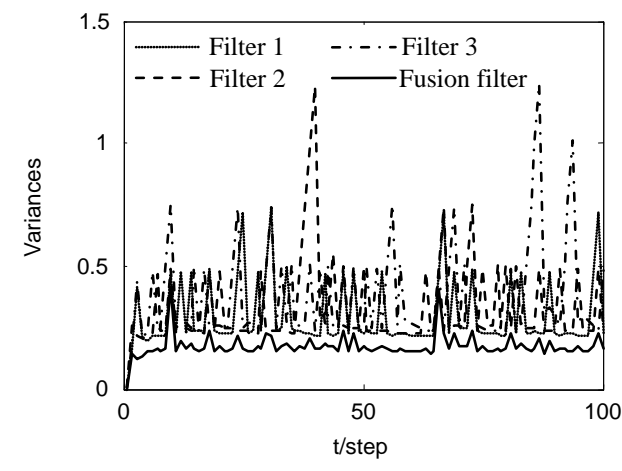

(b) The second-state component

Fig.2. Comparison of filtering error variances

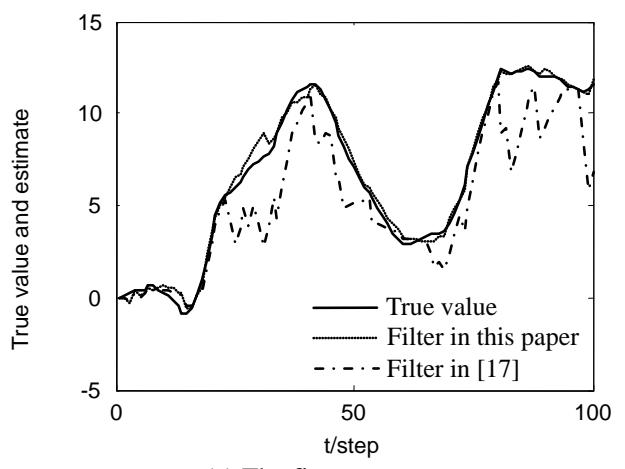

(a) The first-state component

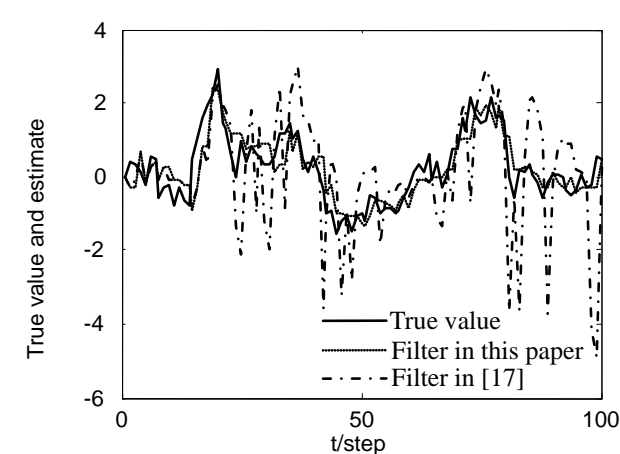

(b) The second-state component

Fig.3. Comparison of tracking performance of the filters in this paper and [17]

Fig.1 gives the tracking performance of the distributed fusion state filter. It is clear that the designed fusion filter has good tracking performance. The filtering error variances of local filters and the distributed fusion filter are shown in Fig.2. We see that the distributed fusion filter has better accuracy than local filters, which verifies the effectiveness of the algorithms in this paper. Fig.3 gives the comparison of our filter and [17] for sensor 1. From Fig.3, we see that our filter has better tracking performance than [17] since packet dropout is not taken into account in [17].

\section{Conclusion}

Distributed fusion filter has been designed for networked systems with packet dropouts and unknown measurement interferences. Without any prior information about the measurement interferences, we have proposed the recursive Kalman-type local state filters independent of unknown interferences for individual sensor subsystems. Based on the local state filters, the estimation error cross-covariance matrices between any two local filters are derived. At last, the distributed fusion filter weighted by matrices is obtained. 


\section{Acknowledgement}

This work was supported in the Chang Jiang Scholar Candidates Program for Heilongjiang Provincial Universities (Project No. 2013CJHB005), and the Province Key Laboratory.

\section{References}

[1] A. B. Soglo, Xianhui Yang. Networked Control System Simulation Design and Its Application[J]. Tsinghua Science and Technology, 2006, 11(3):287-294.

[2] Phillip Lee, Andrew Clark, Linda Bushnell, Radha Poovendran. A Passivity Framework for Modeling and Mitigating Wormhole Attacks on Networked Control Systems[J]. IEEE Transactions on Automatic Control, 2013, 59(12):3224-3237.

[3] Nobuya Takahashi, Masahiro Yokomichi, Osamu Sato. Guaranteed cost control of the networked control system[C]. Sice Conference. IEEE, 2014:626-631.

[4] Keyou You, Lihua Xie. Survey of Recent Progress in Networked Control Systems[J]. Acta Automatica Sinica, 2013, 39(2):101-117.

[5] Nasser E. Nahi. Optimal recursive estimation with uncertain observation[J]. IEEE Transactions on Information Theory, 1969, 15(4):457-462.

[6] Shuli Sun, Lihua Xie, Wendong Xiao, Yeng Chai Soh. Optimal linear estimation for systems with multiple packet dropouts[J]. Automatica, 2008, 44(5):1333-1342.

[7] Jinling Liang, Zidong Wang, Xiaohui Liu. State estimation for coupled uncertain stochastic networks with missing measurements and time-varying delays: the discrete-time case[J]. IEEE Transactions on Neural Networks, 2009, 20(5):781-93.

[8] Shuli Sun, Guanghui Wang. Modeling and estimation for networked systems with multiple random transmission delays and packet losses[J]. Systems \& Control Letters, 2014, 73(73):6-16.

[9] Dong Han, Fanglai Zhu. Simultaneous Estimation of States and Unknown Inputs for Linear Systems Based on Auxiliary Outputs[J]. Acta Automatica Sinica, 2012, 38(6):932-943.

[10] Chien-Shu Hsieh. On the global optimality of unbiased minimum-variance state estimation for systems with unknown inputs[J]. Automatica, 2010, 46(4):708-715.

[11] Chien-Shu Hsieh. State estimation for descriptor systems via the unknown input filtering method[J]. Automatica, 2013, 49(5):1281-1286.

[12] Zhikun Hu, Yan Sun, Bin Jiang, Jing He, et. al. An Optimal Unknown Input Observer Based Fault Diagnosis Method[J]. Acta Automatica Sinica, 2013, 39(8):1225-1230.

[13] Yueyang Li, Maiying Zhong. Fault Detection Filter Design for Linear Discrete Time-varying Systems with Multiple Packet Dropouts[J]. Acta Automatica Sinica, 2012, 41(9):1638-1648.

[14] Chongyan Pang, Shuli Sun. Fusion Predictors for Multisensor Stochastic Uncertain Systems With Missing Measurements and Unknown Measurement Disturbances[J]. IEEE Sensors Journal, 2015, 15(8):4346-4354.

[15] Brian. D. O. Anderson, John. B. Moore, Optimal Filtering[M]. Englewood Cliffs, NJ, USA: Prentice-Hall, 1979.

[16] Shuli Sun, Zili Deng. Multi-sensor optimal information fusion Kalman filter[J]. Automatica, 2004, 40(6):1017-1023.

[17] Jinhua Bai, Shuli Sun. Distributed Fusion Filter for Discrete-time Stochastic Linear Systems with Unknown Sensor Inputs[J]. Science Technology \& Engineering, 2008. 